\title{
Estado da arte das aplicações de curvas de aprendizado
}

\section{The state-of-the-art of learning curve applications}

\author{
Renato Eduardo Stroieke ${ }^{1}$ \\ Flavio Sanson Fogliatto ${ }^{1}$ \\ Michel Jose Anzanello'
}

\begin{abstract}
Resumo: Curvas de aprendizagem permitem representar a melhoria no desempenho de um trabalhador por conta da repetição de um procedimento manual. Este artigo traz uma revisão da literatura das aplicações das curvas de aprendizado. Primeiramente, é apresentada uma breve revisão dos principais modelos de curva de aprendizado. É então apresentada uma revisão das principais utilizações para as curvas de aprendizado, agrupadas em 5 diferentes áreas de pesquisa: ( i) Monitoramento de Custos; (ii) Gerenciamento e Otimização de Produção; (iii) Sequenciamento de tarefas; (iv) Atribuição de Tarefas para Trabalhadores e Balanceamento de Linhas de Produção; e (v) Cálculo do lote econômico de produção com o efeito de aprendizagem.
\end{abstract}

Palavras-chave: Curvas de aprendizado. Programação da produção. Monitoramento de custos.

\begin{abstract}
Learning curves are a graphical representation of an employee performance improvement as a result of task repetition. This paper presents a literature review of the applications of learning curves. Firstly, a brief review of major models of learning curves is presented. Next, a review of the main uses of learning curves is presented grouped into five different areas of research: (i) Cost Monitoring, (ii) Production Management and Optimization, (iii) Task Scheduling, (iv) Assignment of Workers to Tasks and Assembly Line Balancing, and (v) Calculation of Economic Batch Quantity with the learning effect.
\end{abstract}

Keywords: Learning curves. Production programming. Cost monitoring.

\section{Introdução}

As ferramentas da qualidade estão sendo cada vez mais difundidas nas organizações modernas. Técnicas de gerenciamento se tornaram essenciais para a preservação das diversas atividades agregadoras de valor, visto que a competitividade atual limita a entrada de organizações ineficientes no mercado globalizado.

Segundo Ishikawa (1993), só se gerencia aquilo que se mede. Um grande passo para o gerenciamento das pessoas é obter informações que auxiliem na tomada de decisão. Um exemplo é o perfil de aprendizado dos trabalhadores, uma informação que tem ganhado cada vez mais importância no campo da estratégia competitiva (DEMEESTER; QI, 2005). Esse tipo de informação está sendo cada vez mais relevante para que os responsáveis por formação de equipes de trabalho exerçam seu papel de forma eficiente.

No contexto de gerenciamento de produção, o fenômeno de aprendizado refere-se ao aumento de desempenho obtido por repetição da tarefa (BADIRU, 1992; HO; HUANG, 2009). As curvas de aprendizado quantificam a relação entre produção acumulada e a diminuição do custo de produção (DEMEESTER; QI, 2005; YELLE, 1979), observando e prevendo melhorias contínuas no campo fabril e nas organizações de serviços (YANG; WANG; PAI, 2009). O primeiro modelo de curva de aprendizado foi proposto por Wright (1936), por meio de observações na montagem de aviões.

A maioria das pesquisas envolvendo curvas de aprendizado tem por objetivo primário ou secundário aumentar a qualidade da produção pela otimização do desempenho dos processos, da utilização de recursos, e de custos de processamento, entre outros. Alguns trabalhos envolvendo diretamente curvas de aprendizado e qualidade foram realizados por Levy (1965), associando o efeito de aprendizado ao aumento de desempenho de uma organização quando um novo processo é introduzido, e por Li e Rajagopalan (1997), que mostraram que, com a experiência adquirida através do tempo, a empresa produz mais unidades num determinado intervalo de tempo e reduz custos

\footnotetext{
${ }^{1}$ Departamento de Engenharia de Produção, Escola de Engenharia, Universidade Federal do Rio Grande do Sul - UFRGS, Av. Osvaldo Aranha, 99, $5^{\circ}$ andar, CEP 90035-190, Porto Alegre, RS, Brasil, e-mail: renatoes@ producao.ufrgs.br; ffogliatto@producao.ufrgs.br; anzanello@producao.ufrgs.br
} 
de produção, por meio da diminuição do tempo de funcionamento dos equipamentos e de custos materiais.

Alguns trabalhos, envolvendo curvas de aprendizado e qualidade, focam as formulações matemáticas das curvas. Jaber e Guiffrida (2004) introduziram o conceito de "curva de aprendizado da qualidade", analisando dados de retrabalho de itens defeituosos. Ittner, Nagar e Rajan (2001) afirmaram que o aprendizado é função de investimentos em qualidade e processos de repetição. Teng e Thompson (1996) analisaram a qualidade e custos de um novo produto na indústria automotiva, utilizando a curva de aprendizado.

Observando-se o número significativo de publicações abordando o processo de aprendizagem, Anzanello e Fogliatto (2007a) apresentaram uma revisão do estado da arte da literatura sobre curvas de aprendizado, focando na fundamentação teórica e limitações dos principais modelos de curvas de aprendizado. Esse trabalho, por sua vez, visa trazer uma revisão da literatura acerca das principais aplicações das curvas de aprendizado em contextos distintos de produção industrial, como balanceamento de linhas de produção, sequenciamento de tarefas e monitoramento de custos, entre outros. Para tanto, no período de setembro de 2011 até dezembro de 2011, foram pesquisadas publicações referentes a curvas de aprendizado nas bases de dados Science Direct, Emerald e Scielo. Foram utilizadas na pesquisa palavras-chave como "learning curves" e "learning effect". Os artigos citados no corpo desse trabalho foram agrupados conforme as áreas de pesquisa tidas como relevantes pelos autores e com maior concentração de estudos relacionados à ferramenta.

Este artigo apresenta a seguinte estrutura: a seção 2 traz uma breve revisão dos principais modelos de curvas de aprendizado. A seção 3 traz em suas subseções uma revisão das principais linhas de pesquisa sobre curvas de aprendizado. A conclusão do artigo é apresentada na seção 4.

\section{Principais modelos de curvas de aprendizado}

\subsection{Modelos potenciais}

Os modelos de curvas de aprendizado potenciais foram os primeiros a serem criados e os mais utilizados em diversas áreas do conhecimento. Deve-se a Wright (1936) o mérito pela introdução das curvas de aprendizado na Engenharia. Observando a indústria aeronáutica americana, o autor chegou à expressão conhecida como "regra dos 80\%"; nela, para cada duplicação da quantidade de aeronaves produzidas, observava-se uma redução de custos na ordem de $20 \%$. O modelo de Wright é apresentado na Equação 1.

$$
y=C_{1} x^{b}
$$

$\mathrm{Na}$ Equação 1, $y$ indica o tempo necessário para execução da repetição $x$ da tarefa observada, e $C_{1}$ é o tempo de execução da primeira repetição. $O$ parâmetro $b$ indica a velocidade de aprendizado, com valores variando entre 0 e -1 ; quanto mais próximo de -1 , maior é a taxa de aprendizado (ANZANELLO; FOGLIATTO, 2007a; JABER; SIKSTRÖM, 2004).

Com o avanço das pesquisas sobre curvas de aprendizado, a equação de Wright foi sofrendo transformações com o intuído de ser adaptável a diversas situações. O modelo Stanford-b (ANZANELLO; FOGLIATTO, 2007a; BADIRU, 1992) é o modelo de Wright acrescido do parâmetro $B$. A função desse parâmetro adicional é de elevar o patamar de desempenho considerando a experiência prévia do trabalhador. O modelo Stanford-b é apresentado na Equação 2; demais parâmetros mantêm a definição apresentada anteriormente.

$$
\mathrm{y}=C_{1}(x+B)^{b}
$$

A Equação 1 também sofreu alterações, adaptando-se a ambientes que apresentam participação efetiva de maquinário. O modelo de DeJong apresentado na Equação 3 possui o parâmetro $M$, denominado fator de incompressibilidade, que varia de 0 a 1 (ANZANELLO; FOGLIATTO, 2007a); demais fatores permanecem como apresentados anteriormente. Esse parâmetro representa a proporção do tempo total de operação constituído por procedimentos automatizados. Caso o processo em estudo não apresente proporção de atividade influenciada por maquinário, a expressão se reduz ao modelo de Wright, apresentado na Equação 3.

$$
y=C_{1}\left[M+(1-M) x^{b}\right]
$$

O modelo conhecido como curva $\mathrm{S}$, que contempla as premissas do modelo de DeJong e Stanford-b, é apresentado na Equação 4 (ANZANELLO; FOGLIATTO, 2007a).

$$
y=C_{1}\left[M+(1-M)(x+B)^{b}\right]
$$

No modelo de Wright, o tempo de execução da tarefa cai para níveis mínimos quando o número de repetições for muito grande. O modelo Plateau contorna essa dificuldade, apresentando uma constante $C$ que reproduz o desempenho do trabalhador ao atingir o estado estacionário (ANZANELLO; FOGLIATTO, 2007a); demais parâmetros mantêm as definições anteriores. O modelo Plateau é dado na Equação 5.

$$
y=C+C_{1} x^{b}
$$

\subsection{Modelos exponenciais}

A partir dos modelos exponenciais pode-se extrair maior número de informações sobre o processo de 
aprendizado individual. Esses modelos geram previsões de produção mais precisas que as fornecidas pelas curvas potenciais (ANZANELLO; FOGLIATTO, 2007a; NEMBHARD; UZUMERI, 2000). Knecht (1974) introduziu o primeiro modelo de curva de aprendizado exponencial, apresentado na Equação 6. Por meio de uma segunda constante $c$, e utilizando conjuntamente funções exponenciais e potenciais, esse modelo busca aprimorar a modelagem de processos caracterizados por um elevado número de repetições (ANZANELLO; FOGLIATTO, 2007a). Os demais parâmetros possuem o mesmo significado aos das equações anteriores.

$$
y=C_{1} x^{b} e^{c x}
$$

O modelo exponencial de 3 parâmetros é mostrado na Equação 7. Nesse modelo, y representa o desempenho do trabalhador na realização da tarefa, expresso em unidades produzidas após $x$ unidades de tempo de operação; $x$ denota o tempo de operação na tarefa, expresso em unidades de tempo; $k$ é o patamar máximo de desempenho a ser atingido, expresso em número de unidades produzidas por tempo de operação; $p$ designa a experiência prévia do trabalhador na execução da tarefa em questão, expresso em unidades de tempo; e $r$ representa a taxa de aprendizado do trabalhador, expressa em unidades de tempo.

$$
y=k\left(1-e^{-(x+p) / r}\right)
$$

O modelo de tempo constante (TOWILL, 1990) é apresentado na Equação 8.

$$
y=y_{c}+y_{f}\left(1-e^{-\frac{t}{\tau}}\right)
$$

Nesse modelo, $y_{c}$ representa o desempenho inicial do trabalhador (unidades/tempo); e $y_{f}$ indica o patamar máximo de aprendizado realizado pelo trabalhador, também expresso em unidades por tempo. O modelo utiliza o tempo acumulado de operação $(t)$ como variável independente, tendo o mesmo significado do número de unidades produzidas $(x)$ adotado pelos demais modelos de curvas (ANZANELLO; FOGLIATTO, 2007a).

\subsection{Modelos hiperbólicos}

O modelo de curva de aprendizado hiperbólico foi introduzido por Mazur e Hastie (1978). No modelo criado, foi modificada a lógica dos eixos da curva de Wright, fazendo com que as curvas apresentem-se ascendentes em relação ao eixo das abscissas. O modelo hiperbólico de 2 parâmetros, na Equação 9 , tem $y$ como o número de unidades produzidas em um dado intervalo de tempo e $x$ como o intervalo de tempo considerado. Neste modelo, o parâmetro de aprendizado é representado por $r$ e o parâmetro $k$ corresponde ao nível máximo de aprendizado.

$$
y=k\left(\frac{x}{x+r}\right)
$$

Mazur e Hastie (1978) adicionaram o parâmetro $p$, que capta a experiência prévia do trabalhador na execução da tarefa, à Equação 9. Esse novo parâmetro gerou o modelo hiperbólico de três parâmetros, apresentado na Equação 10 (ANZANELLO; FOGLIATTO, 2007a).

$$
y=k\left(\frac{x+p}{x+p+r}\right)
$$

\section{Principais áreas de pesquisa envolvendo o fenômeno de aprendizado}

\section{Monitoramento de custos}

A análise de custos foi o fator gerador do estudo sobre curvas de aprendizado. O primeiro modelo de curva de aprendizado, criado por Wright (1936), mostrava a redução nos custos de produção em uma planta de montagem de aviões na Primeira Guerra Mundial. O autor observou que, com cada duplicação na produção, o custo caía 20\% (ANZANELLO; FOGLIATTO, 2007a; JABER; BONNEY, 1996a; MORRISON, 2008). Alchian (1963) também utilizou dados da indústria bélica americana na previsão dos custos de produção.

$\mathrm{O}$ fato de que os custos de fabricação tendem a seguir uma curva de experiência não se torna útil somente no controle e previsão de custos, implicando também nos preços e lucros (CONLEY, 1970). Altos volumes de produção representam, além da oportunidade para a redução de custos, benefícios trazidos pelo aumento da velocidade do efeito da "curva de experiência", políticas de estoque mais adequadas, racionalização dos processos, melhoria de projetos de trabalho, simplificação de fluxos, etc. (SZWARCFITER; DALCOL, 1997).

Análises de curvas de aprendizado em níveis mais detalhados de planejamento de produção incluem uma redução nos gastos com novos equipamentos, melhor alocação de recursos de trabalho e melhor fluxo de trabalho na fábrica (SMUNT; WATTS, 2003).

O fenômeno de aprendizado é utilizado no monitoramento de custo em diversas áreas da engenharia. Na Engenharia Civil, por exemplo, Leite e Possamai (2001) utilizaram as curvas de aprendizagem para desenvolver um modelo para determinação do índice de diminuição nos tempos gastos para a execução de serviços em setores da construção civil. Segundo a metodologia apresentada, é possível melhor 
determinação dos preços dos serviços e o prazo de execução, sabendo antecipadamente o crescimento de sua produtividade, por intermédio dos índices de diminuição de tempos gerados.

Young II, Masel e Judd (2008) desenvolveram uma metodologia que agrupava perfis de aprendizado utilizando matrizes. O método foi aplicado na previsão de custos em uma indústria de motores. Segundo os autores, os resultados forneceram estimativas muito mais precisas do que os métodos anteriormente disponíveis, que utilizavam a média ponderada das taxas individuais de aprendizagem.

Outros autores, como Neuhaus e Bunke (2007), também utilizaram o fenômeno de aprendizado para estimar custos de uma técnica de edição gráfica. Pereira e Suslick (2003) utilizaram a curva de Stanford - B para prever custos em uma indústria de mineração; os custos previstos divergiram dos reais em apenas $10 \%$.

Plaza e Rohlf (2008) demonstraram que a combinação de treinamento formal com treinamento no posto de trabalho minimiza custos com consultorias contratadas no apoio a projetos. Nadeau et al. (2010), estudando aprendizado em uma indústria automotiva, concluíram que a variação na aprendizagem está ligada à estrutura individual do processo de custo.

Nemet (2006), estudando a redução dos custos das lâmpadas fotovoltaicas nos últimos anos, concluiu que o fenômeno de aprendizado não contribuiu sozinho para que essa redução ocorresse. Fatores como tamanho da planta, eficiência da produção e o custo de silício apresentaram grande interação com a redução dos custos de produção das lâmpadas.

Estudos recentes utilizam a estrutura dos modelos de aprendizagem para prever a evolução nos custos de produção de biocombustíveis e energias renováveis em um futuro próximo. De Wit et al. (2010), investigando o processo de aprendizado, afirmam que o potencial de redução nos custos de instalação de novas plantas entre 2005 e 2030 é consideravelmente maior do que da primeira geração de biocombustíveis. Tsuchiya e Kobayashi (2004) estimaram o custo de produção de membranas de células combustíveis à base de paládio e prata, utilizadas em automóveis, a partir de curvas de aprendizado. Segundo essa pesquisa, com o advento da produção em massa desse tipo de produto, o custo das células pode se equiparar ao custo dos motores atuais. Analisando os custos de produção de células combustíveis, Schoots, Kramer e van der Zwaan (2010) chegaram à conclusão de que, apoiada pelo processo de aprendizado, a redução foi em média de $80 \%$ entre cada um dos períodos analisados. Broek et al. (2009) ampliaram o conceito de curvas de aprendizado para simultaneamente considerar melhorias nos parâmetros de desempenho do sistema, bem como variáveis de custo, objetivando avaliar o futuro desenvolvimento de usinas de energia com captura de $\mathrm{CO}_{2}$.
Bake et al. (2009) demonstraram, utilizando modelagem de curvas de aprendizado, que o custo de matéria-prima, assim como o custo de produção de etanol no Brasil, está caindo no decorrer dos anos. Segundo suas estimativas, o custo de produção pode cair de aproximadamente $340 \mathrm{US} \$ / \mathrm{m}^{3}$, na época da realização do trabalho, para algo entre 200 e 260 US $\$ / \mathrm{m}^{3}$ em 2020.

Analisando o setor energético, Kahouli-Brahmi (2008) dividiu os estudos de curva de aprendizado em dois modelos: os modelos bottom-up, cuja modelagem fornece novos conhecimentos em relação às perspectivas de opções tecnológicas e seu potencial na redução de custos, e os modelos top-down, que utilizam esse conhecimento para análise das considerações estratégicas, especialmente voltados à inovação e processo de difusão de energia.

Weitzel, Rovere e Cunha (2006) estudaram o comportamento dos custos futuros das tecnologias renováveis em comparação às fósseis no intervalo 2005-2020, utilizando a curva de aprendizado. O objetivo principal desse trabalho foi contribuir com o planejamento energético, de modo a promover o aumento da participação das fontes alternativas na matriz energética brasileira.

\subsection{Gerenciamento e otimização de produção}

Entender os fatores que afetam o aprendizado organizacional permite que gerentes aumentem o desempenho das organizações em diferentes áreas (ARGOTE; EPPLE, 1990; TARNANIDIS; PAPATHANASIOU; FILAKIS, 2006). Diversos autores utilizaram os modelos mais tradicionais de curvas de aprendizado (destacadamente os potenciais e hiperbólicos) para aperfeiçoar pontos específicos de processos ou linhas de produção. Isso se dá, por exemplo, em Chatzimichali e Tourassis (2007), que estudaram o efeito de aprendizado em uma indústria de telhas cerâmicas, e em Plaza, Ngwenyama e Rohlf (2010), que mostraram como a teoria de curvas de aprendizado pode melhorar a gestão de projetos de implementação de novas tecnologias, utilizando dados de implementação de projetos ERP (Enterprise Resource Planning).

Outros autores que estudaram o efeito de aprendizado em projetos de desenvolvimento de software foram Pendharkar e Subramanian (2007). Foi estudada a relação entre o esforço do programador e sua experiência na ferramenta "engenharia de software assistida por computador integrado" (ICASE), mostrando que essa relação pode ser descrita por uma curva de aprendizado. Ngwenyama, Guergachi e McLaren (2007), também estudando a aplicação de curva de aprendizado na fabricação de softwares, chegaram à conclusão de que a troca de atividade 
constante faz com que o trabalhador não obtenha ganhos de produtividade. $\mathrm{O}$ trabalho desenvolvido buscou encontrar o tempo de substituição da tarefa que maximiza os ganhos de produtividade da organização.

Adler (1990) estudou a evolução da produtividade em uma empresa de eletrônicos, chegando à conclusão de que o aprendizado compartilhado é fator crítico na melhoria de produtividade, bem como no desenvolvimento de produto, na interface de manufatura, no posicionamento primário da planta e no aprendizado contínuo.

Os processos organizacionais por trás da evolução da curva de aprendizado no primeiro ano de operação de uma nova fábrica de automóveis foram ilustrados por Prochno (2004). Foi sugerido que a curva de aprendizado global é de fato o resultado de um processo de integração que reúne várias curvas de aprendizado individuais em diferentes áreas da organização.

Wiersma (2007) concluiu que variações nas taxas de aprendizagem são explicadas pelo percentual de empregados temporários trabalhando em uma unidade fabril, pela capacidade de fabricação, pelo grau de heterogeneidade do produto e pela quantidade de tempo gasta em outras tarefas. Stan e Vermeulen (2010) mostraram que o aprendizado organizacional é maior em empresas que apresentam tarefas com maior grau de dificuldade na execução.

Alguns gerentes de produção deixam de utilizar dados de curva de aprendizado no planejamento de produção, pois, muitas vezes, os dados de aprendizado individual apresentam grande variabilidade. Smunt e Watts (2003) estudaram esse tipo de problema e concluíram que, aplicando métodos de agregação simples, é possível melhorar a precisão das taxas de aprendizagem. Shafer, Nembhard e Uzumeri (2001), estudando como os padrões de aprendizado e esquecimento afetam o desempenho operacional de uma linha de montagem de rádios para automóveis, concluíram que a modelagem da tendência central, desconsiderando o perfil de aprendizado individual dos trabalhadores, leva a subestimar sistematicamente a produtividade global.

Fioretti (2007) afirmou que, estudando a curva de aprendizado, é possível obter os parâmetros de uma equação de aprendizado, porém não vinculá-los a propriedades específicas de uma organização. Mais recentemente, Fioretti (2009) afirmou que existe uma dificuldade na utilização de curva de aprendizado no gerenciamento de produção, pois elas acabam fornecendo "um palpite" sobre o comportamento futuro, ou seja, podendo ocorrer de nenhuma curva individual de aprendizado ser equivalente à sua curva teórica.

Os fatores que influenciam no aprendizado também foram estudados por Balasubramanian e Lieberman (2010), que concluíram que a intensidade de aprendizado está fortemente ligada ao desempenho da empresa. As empresas com maior taxa de aprendizado são as que mais investem em pesquisa e desenvolvimento, em publicidade, e possuem maior capacidade do capital empregado. Argote e Epple (1990) estudaram os fatores que fazem com que ocorram variações no processo de aprendizado em diferentes organizações. Os fatores encontrados foram o esquecimento, a troca de trabalhadores, a transferência de conhecimento entre diferentes produtos e diferentes organizações, transferência incompleta dentro da organização e economia de escala.

Pruett e Thomas (2008) mostraram que, quanto maior a experiência em inovação da empresa, menos traumático será o processo de aprendizagem na produção. Como uma empresa ganha experiência na transferência de aprendizado entre inovações sucessivas, o aprendizado em novos produtos pode ser menor.

A existência do fenômeno de aprendizado também foi estudada no setor de serviços. Boone e Ganeshan (2001) encontraram uma relação significativa entre a experiência na produção e produtividade, comprovando a existência do processo de aprendizagem nesse tipo de setor.

\subsection{Sequenciamento de tarefas com o efeito de aprendizado}

A ordem de execução das tarefas em um setor produtivo deve seguir um conjunto de regras de acordo com o objetivo da empresa, tais como reduzir o atraso das entregas, atender a pedidos prioritários, diminuir o tempo de produção, aumentar a utilização dos recursos e reduzir o estoque em processo (LOPES, 2008).

Em problemas de programação tradicional, a maioria das pesquisas pressupõe que todos os trabalhos apresentam tempos de processamento constante. Em especial, os tempos de processamento de trabalho são considerados independentes de sua posição na sequência de programação (EREN; GÜNER, 2007); ou seja, nesse tipo de problema, geralmente é desprezado o processo de aprendizagem, no qual o tempo de execução da tarefa vai diminuindo com o número de repetições. Esse fenômeno é bem observado em linhas de montagem nas quais ocorrem atividades manuais mais intensas, mas também ocorre em processos mais automatizados, nos quais os tempos de setup de equipamentos, ou seja, tempo de ajuste dos equipamentos para a produção de novos itens, vão diminuindo com o número de repetições.

Os pesquisadores que estudam o problema do sequenciamento de tarefas vinculado ao processo de aprendizagem costumam modelar o tempo de execução da tarefa de dois modos: 
Aprendizado baseado na posição: o tempo de execução da tarefa depende somente da sua posição no sequenciamento global de tarefas (BISKUP, 2008).

Aprendizado baseado na soma de tempos de processamento: o tempo de execução da tarefa está relacionado com o tempo de processamento dos trabalhos processados até o momento (BISKUP, 2008). Essa abordagem é geralmente utilizada quando há grande interação humana durante o processamento.

O primeiro autor a considerar o processo de aprendizagem no sequenciamento de tarefas foi Biskup (1999), utilizando o aprendizado baseado na posição. Nesse tipo de problema, o modelo de aprendizado utilizado é uma variação do modelo de Wright (1936), representado pela Equação 11:

$$
p_{i r}=p_{i} r^{a}
$$

em que $p_{i r}$ é o tempo de processamento do trabalho $i$ alocado na posição $r$, e $a \leq 0$ é a constante de aprendizado.

Na obtenção do tempo de execução da tarefa, foi considerado o número de repetições já realizadas. Foram dois os objetivos para a solução desse problema: (i) minimizar o desvio na data de entrega, ou seja, diminuir o custo de ter uma tarefa sendo entregue muito tarde ou muito cedo; e (ii) minimizar o tempo de fluxo.

Lee e Wu (2004) objetivaram minimizar o tempo de execução das tarefas quando se leva em consideração o agendamento em duas máquinas. Eles consideraram que o processo de aprendizagem ocorre separadamente entre as duas máquinas. Resultados computacionais mostraram que o algoritmo branch-and-bound apresenta um bom desempenho na resolução desse tipo de problema quando se trabalha com menos de 30 tarefas.

Mani, Chang e Chen (2009) propuseram uma metodologia em que a sequência das tarefas é feita utilizando o algoritmo de Aneja e Nair (1979), capaz de resolver problemas de transporte que possuem dois objetivos distintos. No entanto, enquanto o algoritmo original buscava encontrar um caminho mais eficiente para o problema de distribuição, a adaptação realizada faz com que seja obtido o sequenciamento de tarefas desejado. O objetivo desse trabalho foi encontrar um sequenciamento que minimiza o tempo total de execução da tarefa.

Eren e Güner (2007) estudaram o processo de sequenciamento de tarefas em uma única máquina, com o objetivo de minimizar o atraso total de produção. Foi desenvolvido um modelo de programação inteira para resolver o problema, que se mostrou eficiente para o sequenciamento de até 25 tarefas.

Eren (2009) formulou o problema de sequenciamento de trabalhos de modo a incluir o aprendizado nos tempos de setup e de remoção do material presente na operação. O objetivo de minimizar o tempo de conclusão do trabalho e, consequentemente, diminuir o atraso na entrega foi atingido quando se consideraram até 15 tarefas e 5 máquinas.

Kuo e Yang (2007) utilizaram o conceito de aprendizado baseado na soma de tempos de processamento no cálculo do sequenciamento de tarefas. O modelo de aprendizado sugerido pelos autores vem apresentado na Equação (12).

$$
p_{i r}=\left(1+p_{[1]}+p_{[2]}+p_{[r-1]}\right)^{a}
$$

em que $p_{i r}$ é o tempo atual de processamento da atividade $J_{i}$ alocada na posição $r$; e $a$ é a constante de aprendizado. Pode-se observar que o tempo atual de execução da tarefa é afetado por todas as $(r-1)$ tarefas agendadas. O objetivo desse trabalho foi encontrar uma sequência de tarefas que minimize o tempo total de operação em uma única máquina.

Koulamas e Kyparisis (2008) consideraram o problema de sequenciamento com tempo de setup proporcional à duração dos trabalhos já sequenciados. Foi introduzido no problema um termo que captura a dependência entre o tempo de setup e a sequência pregressa de trabalhos já sequenciados (PSD - Past sequence dependent setup time). Kuo e Yang (2007) consideraram o efeito de aprendizagem no sequenciamento em uma única máquina, considerando os tempos de setup proporcional à duração dos trabalhos já sequenciados, e o processo de aprendizagem na execução da tarefa. A função objetivo foi definida de modo a minimizar valores como o de makespan, ou seja, o tempo total de produção.

Janiak et al. (2009) apresentaram um modelo de sequenciamento com o objetivo de diminuição do makespan utilizando o modelo de aprendizado em formato de $S$. Para resolver o problema, foram utilizados o método Branch and Bound e algoritmos heurísticos rápidos. Recentemente, Lee (2011) propôs um novo modelo de aprendizado, no qual a curva de aprendizado também apresenta formato de $S$, e utilizou esse modelo no sequenciamento de tarefas. Para problemas de minimização do atraso no tempo de produção em uma única máquina, o autor demonstrou que o problema é polinomialmente solucionável. Bai, Wang e Wang (2012) apresentaram uma metodologia de sequenciamento de tarefas em uma única máquina, considerando um modelo de aprendizado do tipo exponencial.

Wang, Sun e Sun (2010) consideraram o aprendizado baseado na soma de tempos de processamento como uma função exponencial para o sequenciamento de tarefas em uma única máquina. $\mathrm{O}$ trabalho provou que o problema de minimização do makespan, de minimização do tempo total de produção e de minimização da soma quadrática dos tempos de processamento pode ser resolvido pela regra que consiste em alocar o trabalho com menor tempo de 
processamento à frente dos demais (SPT - smallest processing time first).

Lee e Lai (2011) estudaram o problema de sequenciamento de tarefas em uma máquina considerando o efeito de aprendizagem, que diminui o tempo de execução da tarefa com o passar do tempo, e o efeito de deterioração, que apresenta o comportamento oposto. Conforme Browne e Yechiali (1990), o processo de deterioração em problemas de sequenciamento de tarefas é modelado conforme a Equação 13:

$$
p_{j}^{a}=p_{j}+\alpha_{j} t
$$

em que $p_{j}$ é o tempo de processamento normal da tarefa; $\alpha$ é a taxa de deterioração; e $t$ é o tempo de início da tarefa $J_{i}$.

Yin, Xu e Wang (2010) estenderam o trabalho de sequenciamento em uma única máquina, considerando o tempo de execução da tarefa tanto como função de sua posição no sequenciamento, como também função do tempo de processamento dos trabalhos processados até o momento. O objetivo do problema foi reduzir o makespan e o atraso da realização do trabalho.

Yin e Xu (2011) estenderam o trabalho de sequenciamento de tarefas com aprendizado e deterioração, apresentando uma generalização significativa dos modelos apresentados na literatura até então, trabalhando-se com uma única máquina.

Anzanello e Fogliatto (2010) apresentaram uma metodologia de sequenciamento de tarefas com o objetivo de minimizar o desvio total da data de entrega prometida para os trabalhos. Cada trabalhador foi considerado como uma máquina paralela não relacionada, cujos dados foram coletados e modelados utilizando curvas de aprendizado. Os autores aplicaram sua metodologia em uma fábrica de calçados por ser um ambiente de grande variedade.

Wu, Yin e Cheng (2011) introduziram o problema de sequenciamento de tarefas em uma única máquina com o efeito de aprendizado, utilizando um modelo de aprendizado truncado. Segundo os autores, em modelos convencionais, o tempo de execução da tarefa cai a zero quando são considerados lotes grandes. Para resolver esse problema, foi adicionado um parâmetro de controle no cálculo do tempo de execução da tarefa.

Yang (2011) estendeu os trabalhos de sequenciamento de tarefas em uma única máquina com efeitos de aprendizagem e deterioração considerando agrupamentos de produtos. Em sua formulação, os produtos eram agrupados em família de acordo com suas similaridades para então ser processados.

Hsu, Kuo e Yang (2011) formularam o problema de sequenciamento de tarefas com aprendizado baseado nas execuções anteriores em uma única máquina e com efeito de aprendizado considerando máquinas paralelas e não correlacionadas. Os autores mostraram que o problema de minimização do tempo total de processamento da tarefa pode ser resolvido em tempo polinomial.

\subsection{Atribuição de tarefas a trabalhadores e balanceamento de linhas de produção}

A atribuição de tarefas a trabalhadores é uma etapa muito importante no gerenciamento da produção. Os estudos atuais nessa área trazem benefícios potenciais, tais como: (1) auxílio na elaboração de simulações de produção com força de trabalho heterogênea; (2) criação de estimativas de produção para novos produtos, cujas tarefas são mais complexas que as do passado; (3) inclusão de características individuais nos sistemas de alocação de tarefas (NEMBHARD; OSOTHSILP, 2002).

Anzanello e Fogliatto (2005) propuseram um método para alocação de tarefas a trabalhadores, que foi aplicado em uma indústria calçadista. Os produtos foram inicialmente agrupados em famílias, de acordo com a similaridade de demanda relativamente à operação de costura. Esses produtos foram então manufaturados por três grupos de trabalhadores. $\mathrm{O}$ modelo que melhor adequou-se aos dados de aprendizado coletados foi o hiperbólico. Os parâmetros dos modelos obtidos foram analisados por dois métodos distintos: análise isolada do parâmetro $r \mathrm{e}$ integração das curvas de aprendizagem, permitindo apontar a equipe mais habilitada à execução das operações, de acordo com a duração da corrida de produção. Os autores concluíram que a regressão mostrou-se adequada para a alocação de produtos a equipes em corridas de produção de curta duração. A integração, por sua vez, mostrou-se mais robusta que a regressão para fins de alocação, podendo ser utilizada em corridas de durações distintas mediante a comparação das áreas geradas pelas curvas de aprendizagem. Em Anzanello e Fogliatto (2007b), o trabalho foi estendido tal que o processo de alocação de tarefas para trabalhadores em uma linha de montagem com diferentes tempos de setups foi investigado. Os produtos a serem processados foram agrupados em três famílias, de acordo com sua similaridade, e designados para times de trabalhadores. Os dados de desempenho foram então coletados e a alocação de atividades foi realizada por meio de dois métodos analíticos: um para corridas de produção de curta duração e outro para corridas de longa duração.

O fenômeno de desmotivação na alocação de tarefas para o trabalhador foi analisado por Azizi, Zolfaghari e Liang (2010). Foi demonstrado que, depois de certo período de tempo no posto de trabalho, é necessário que o trabalhador mude de atividade, a fim de não prejudicar seu desempenho. Um benefício da rotação de atividades é o aumento da satisfação 
do trabalhador (CUNNINGHAM; EBERLE, 1990; DAVIS; TAYLOR, 1979). Porém, com a mudança de atividade surge um novo período de aprendizagem, no qual o desempenho do trabalhador ainda não é pleno, gerando perdas para o processo. Azizi, Zolfaghari e Liang (2010) apresentam uma formulação matemática que minimiza as perdas de produtividade devidas à rotação de trabalhadores.

Cohen, Vitner e Sarin (2006) trataram do problema de alocação de trabalhadores em uma linha de montagem, objetivando minimizar o makespan de um lote de produtos com baixa demanda global. Foi pressuposta a não existência de buffers entre os postos de trabalho, além de taxa de aprendizado constante em toda a linha de produção. Foi demonstrado que, mediante tais suposições, a distribuição desigual de trabalho nas estações é melhor do que a distribuição equilibrada para a minimização do makespan. Esse trabalho foi estendido por Cohen et al. (2008a), que consideraram as variações de aprendizado em sua formulação, e por Cohen (2008b), que incluiu a existência de buffers entre os postos de trabalho, considerando três diferentes padrões de aprendizado.

Otero et al. (2009) criaram uma metodologia de alocação de tarefas à trabalhadores utilizando modelagem de aprendizagem em projetos de software, para casos em que os conjuntos de habilidades ideais não estão disponíveis. Corominas, Olivella e Pastor (2010) estudaram a alocação de atividades a um grupo de trabalhadores juntamente com o agendamento dessas atividades. A formulação apresentada tinha por objetivo reduzir o tempo de execução das tarefas. Guimarães, Anzanello e Renner (2012) obtiveram uma redução de $80 \%$ no número de acidentes e de $45,65 \%$ no índice de absenteísmo em uma indústria de calçados, utilizando uma metodologia de atribuição de tarefas, integrando a curva de aprendizado com o método de Análise Macroergonômica do Trabalho.

Toksari et al. (2008) introduziram o conceito de aprendizado em problemas de balanceamento de linhas de montagem simples e em U, objetivando minimizar o número de estações de trabalho. Toksari et al. (2010) estenderam o trabalho inicial tal que, além do aprendizado, fosse considerada também a deterioração dos produtos.

\subsection{Lote econômico de produção com o efeito de aprendizagem}

O lote econômico é calculado pela Equação 14.

$$
\mathrm{Q}^{*}=\sqrt{\frac{2 R P}{i C}}
$$

em que Q* é o tamanho do lote econômico de fabricação; $R$ é a demanda anual do item em questão; $P$ é o custo de setup para produção do item; $i$ é a taxa de guarda anual do estoque; e $C$ é o preço unitário de fabricação do item. O cálculo do tamanho ótimo de lote de produção pressupõe custos de produção constantes, o que não ocorre durante o processo de aprendizagem (JABER; BONNEY, 2007). A premissa de taxa de produção constante também não é válida quando o operador começa a produzir um novo produto, troca de máquina, recomeça a atividade após paradas ou utiliza uma nova técnica de produção (JABER; BONNEY, 1999).

O cálculo do lote econômico de produção considerando o processo de aprendizagem foi introduzido por Keachie e Fontana (1966). Desde então, problemas associados ao seu cálculo vêm sendo explorados na literatura.

Num cálculo do tamanho de lote para certo período de produção são necessárias informações muito precisas dos custos de setup. Visto que essas informações são de difícil obtenção, Rachamadugu e Tan (1997) apresentaram uma metodologia para calcular o tamanho de lote ótimo quando os custos de setup diminuem monotonicamente devido ao aprendizado. Segundo os autores, a metodologia apresentada se mostrou eficiente para o cálculo do tamanho de lote ótimo em ambientes que apresentam uso intensivo de maquinário e altas taxas de aprendizado.

Jaber e Bonney (1996b) calcularam o lote econômico de produção partindo do modelo de aprendizado de De Jong, que impõe um limite no processo de aprendizagem, evitando que o custo de produção caia para zero num futuro indefinido. Os autores utilizaram também uma aproximação da estimativa do custo de guarda desenvolvida por Fisk e Ballou (1982). Jaber e Bonney (2007) calcularam o lote econômico de produção incluindo o efeito de esquecimento e considerando o aprendizado dependente do número de unidades já produzidas. $\mathrm{O}$ modelo de esquecimento utilizado no trabalho foi o mesmo proposto por Jaber e Bonney (1996a).

Rachamadugu e Schriber (1995) desenvolveram dois procedimentos heurísticos para a determinação do tamanho de lote econômico envolvendo o processo de aprendizagem. O primeiro procedimento, que utiliza o custo atual de setup, se mostrou mais apropriado em ambientes em que a aprendizagem ocorre de modo mais ágil. O segundo procedimento, que utiliza o menor valor de setup registrado, é mais apropriado para ambientes com baixo ritmo de aprendizagem.

Jaber e Bonney (2001) compararam o problema de cálculo de lote econômico de produção com o efeito de aprendizado com outro em que a taxa de diminuição de tempo de operação é constante. Os autores chegaram à conclusão de que, embora as duas situações indiquem a produção de lotes menores, os custos totais de produção não são significantemente afetados. 
Jaber e Bonney (2003) estudaram o efeito de aprendizado no cálculo do lote de produção em um ambiente que apresentava não só o aprendizado, mas também o processo de esquecimento nos setups e no retrabalho dos produtos. Os autores consideraram que o efeito de aprendizagem também ocorre no retrabalho dos produtos e que a deterioração dos produtos e o esquecimento aumentam com as interrupções nos processos. Os resultados mostraram que, enquanto a aprendizagem e esquecimento no setup indicaram a produção de lotes menores, a aprendizagem no retrabalho do produto indicou a produção de lotes maiores.

Balkhi (2003) propôs um modelo de cálculo do lote ótimo de produção seguindo as seguintes condições: taxa de demanda e taxa de deterioração são funções do tempo; itens deteriorados enquanto produzidos são armazenados e a escassez é permitida. Primeiramente foi calculado o lote econômico de produção para as dadas condições excluindo-se o processo de aprendizado. Posteriormente, o problema foi reformulado incluindo-se o processo de aprendizado.

Alamri e Balkhi (2007) estudaram o efeito de aprendizagem e esquecimento no cálculo do lote econômico de produção, incluindo ainda na formulação o processo de deterioração e de demanda variando em função do tempo. Foi afirmado que a demanda variando com o tempo faz com que em cada ciclo sejam produzidos lotes menores.

Chen, Lo e Liao (2008) calcularam o tamanho do lote econômico de produção considerando o processo de aprendizado e o sistema de produção imperfeito, ou seja, permitindo escassez de produção. O objetivo do trabalho foi encontrar o menor custo de produção por meio do cálculo do lote econômico de produção e da taxa de escassez em cada ciclo de produção. Chiu e Chen (2005) modelaram o problema de lote econômico de produção considerando os efeitos de aprendizagem e de esquecimento tanto na produção como no setup. Foi demonstrado que o processo de aprendizado na produção influencia mais intensamente no cálculo do lote econômico de produção do que o aprendizado no setup.

Jaber, Goyal e Imran (2008) apresentaram uma formulação matemática para o cálculo do lote econômico de produção considerando a redução na incidência de itens avariados na produção como decorrência do aprendizado. O modelo foi validado em uma indústria automotiva.

Jaber, Bonney e Moualek (2009a) incluíram o conceito termodinâmico de entropia ao cálculo do lote econômico de produção, considerando o processo de aprendizagem e de esquecimento. Os autores concluíram que, enquanto o aprendizado direciona a um menor tamanho de lotes de produção, o esquecimento e a entropia apresentam um efeito reverso, aumentando o tamanho dos lotes. Jaber et al. (2009b) estenderam o conceito de entropia no cálculo de tamanho ótimo de lotes para produção com itens deteriorados. Os autores sugeriram ser melhor aumentar o fluxo de mercadorias por meio de descontos nos preços do que reduzir o custo para recuperar as perdas de receitas devido à deterioração, em ambientes de rápida deterioração.

Wahab e Jaber (2010) estudaram uma formulação para lote econômico de produção com processo de aprendizado, considerando itens com qualidade imperfeita e diferentes custos de guarda. Os autores mostraram que, ao desconsiderar o processo de aprendizado, o tamanho do lote de produção aumenta proporcionalmente com o número de itens deteriorados. Quando o efeito de aprendizado é considerado, inicialmente o tamanho do lote de produção é maior do que um que possua o mesmo custo de guarda; contudo, concluído o aprendizado no sistema, a diferença entre tamanho de lotes com e sem diferentes custos de guarda e itens defeituosos desaparece.

Khan, Jaber e Wahab (2010) formularam o lote econômico de produção considerando o processo de aprendizagem na inspeção dos produtos. Foram estudadas três situações quanto ao aprendizado dos trabalhadores: na primeira, o trabalhador inicia cada processo de inspeção sem possuir conhecimento prévio; na segunda, o trabalhador não apresenta perda de conhecimento com as mudanças de produção; na terceira, há perda parcial de conhecimento com as mudanças de produção.

Recentemente, Jaber e El Saadany (2011) apresentaram um modelo de cálculo econômico de produção que contempla o aprendizado na produção e na remanufatura (reparos na produção). Foi concluído que existe um limite nos investimentos em aprendizado dos trabalhadores que pode trazer economia, ou seja, o aprendizado pode ser acelerado até certo limite; depois disso, passa a ser uma aplicação não justificada de recursos.

\section{Conclusões}

O processo de aprendizagem é aquele em que um trabalhador adquire experiência na realização de certa tarefa, diminuindo o tempo de execução da tarefa posterior. As curvas de aprendizado, que modelam o processo de aprendizagem, têm se mostrado uma ferramenta com várias utilidades em Engenharia de Produção desde que Wright (1936) desenvolveu o primeiro modelo de curva de aprendizado.

Este artigo apresentou uma revisão da literatura sobre as principais utilizações das curvas de aprendizado. Primeiramente foi apresentada uma breve revisão dos principais modelos de curva de aprendizado. Na sequência, foi apresentada uma revisão das principais utilizações para as curvas 
de aprendizado: Monitoramento de Custos; Gerenciamento e Otimização de Produção; Sequenciamento de tarefas; Atribuição de Tarefas para Trabalhadores e Balanceamento de Linhas de Produção; e Cálculo do lote econômico de produção com o efeito de aprendizagem.

A revisão de literatura apresentada neste trabalho traz contribuições gerenciais significativas, à medida que mostra como o processo de aprendizado interfere diretamente no Planejamento e Controle de Produção, assim como nos custos totais de produção.

Futuras pesquisas sobre curvas de aprendizado podem abordar a modelagem de aprendizado coletiva, visto que, na maioria dos estudos, é utilizado o processo individual de aprendizado. Outras pesquisas podem utilizar a curva de aprendizado para a criação de agrupamentos homogêneos de trabalhadores, ou ainda, estudar como o processo de aprendizado contribui na definição de estratégias da empresa por afetar diretamente os custos de produção.

\section{Referências}

ADLER, P. S. Shared Learning. Management Science, v. 36, n. 8, p. 938-957, 1990. http://dx.doi.org/10.1287/ mnsc. 36.8 .938

ALAMRI, A. A.; BALKHI, Z. T. The effects of learning and forgetting on the optimal production lot size for deteriorating items with time varying demand and deterioration rates. International Journal of Production Economics, v. 107, n. 1, p. 125-138, 2007. http://dx.doi. org/10.1016/j.ijpe.2006.08.004

ALCHIAN, A. Reliability of Progress Curves in Airframe Production. Econometrica, v. 31, n. 4, p. 679-693, 1963. http://dx.doi.org/10.2307/1909166

ANEJA, Y. P.; NAIR, K. P. K. Bicriterion transportation problem. Management Science, v. 25, n. 1, p. 73-78, 1979. http://dx.doi.org/10.1287/mnsc.25.1.73

ANZANELLO, M. J.; FOGLIATTO, F. S. Alocação de modelos de produtos a equipes de trabalhadores baseada em modelos de curvas de aprendizagem. Revista Produção, v. 15, n. 2, p. 221-234, 2005.

ANZANELLO, M. J.; FOGLIATTO, F. S. Curvas de aprendizado: estado da arte e perspectivas de pesquisa. Gestão \& Produção, v. 14, n. 1, p. 109-123, 2007 a. http://dx.doi.org/10.1590/S0104-530X2007000100010

ANZANELLO, M. J.; FOGLIATTO, F. S. Learning curve modelling of work assignment in mass customized assembly lines. International Journal of Production Research, v. 45, n. 13, p. 2919-2938, 2007b. http:// dx.doi.org/10.1080/00207540600725010

ANZANELLO, M. J.; FOGLIATTO, F. S. Scheduling learning dependent jobs in customised assembly lines. International Journal of Production Research, v. 48, n. 22, p. 6683-6699, 2010. http://dx.doi. org/10.1080/00207540903307599

ARGOTE, L.; EPPLE, D. Learning Curves in Manufacturing. Science, v. 247, n. 4945, p. 920-924, 1990. PMid:17776451. http://dx.doi.org/10.1126/ science.247.4945.920
AZIZI, N.; ZOLFAGHARI, S.; LIANG, M. Modeling job rotation in manufacturing systems: The study of employee's boredom and skill variations. International Journal of Production Economics, v. 123, n. 1, p. 69-85, 2010. http://dx.doi.org/10.1016/j. ijpe.2009.07.010

BADIRU, A. B. Computational Survey of Univariate and Multivariate Learning Curve Models. IEEE Transactions on Engineering Management, v. 39, n. 2, p. 176-188, 1992. http://dx.doi.org/10.1109/17.141275

BAI, J.; WANG, M.-Z.; WANG, J.-B. Single machine scheduling with a general exponential learning effect. Applied Mathematical Modelling, v. 36, n. 2, p. 829-835, 2012. http://dx.doi.org/10.1016/j. apm.2011.07.002

BAKE, J. V. et al. Explaining the experience curve: Cost reductions of Brazilian ethanol from sugarcane. Biomass and Bioenergy, v. 33, n. 4, p. 644-658, 2009. http:// dx.doi.org/10.1016/j.biombioe.2008.10.006

BALASUBRAMANIAN, N.; LIEBERMAN, M. B. Industry Learning Environments and the Heterogeneity of Firm Performance. Strategic Management Journal, v. 31, n. 4, p. 390-412, 2010.

BALKHI, Z. T. The effects of learning on the optimal production lot size for deteriorating and partially backordered items with time varying demand and deterioration rates. Applied Mathematical Modelling, v. 27, n. 10, p. 763-779, 2003. http://dx.doi.org/10.1016/ S0307-904X(03)00081-7

BISKUP, D. Single-machine scheduling with learning considerations. European Journal of Operational Research, v. 115, n. 1, p. 173-178, 1999. http://dx.doi. org/10.1016/S0377-2217(98)00246-X

BISKUP, D. A state-of-the-art review on scheduling with learning effects. European Journal of Operational Research, v. 188, n. 2, p. 315-329, 2008. http://dx.doi. org/10.1016/j.ejor.2007.05.040

BOONE, T.; GANESHAN, R. The effect of information technology on learning in professional service organizations. Journal of Operations Management, v. 19, n. 4, p. 485-495, 2001. http://dx.doi.org/10.1016/ S0272-6963(00)00064-4

BROEK, M. V. et al. Effects of technological learning on future cost and performance of power plants with CO2 capture. Progress in Energy and Combustion Science, v. 35, n. 6, p. 457-480, 2009. http://dx.doi. org/10.1016/j.pecs.2009.05.002

BROWNE, S.; YECHIALI, U. Scheduling deteriorating jobs on a single processor. Operations Research, v. 38, n. 3, p. 495-498, 1990. http://dx.doi.org/10.1287/ opre.38.3.495

CHATZIMICHALI, A. P.; TOURASSIS, V. D. An Empirical Investigation of Learning Curve Laws in the Tile Manufacturing Industry. In: IEEE INTERNATIONAL CONFERENCE ON INDUSTRIAL ENGINEERING AND ENGINEERING MANAGEMENT, 2007, Singapura. Proceeding... Singapura, 2007. p. 1110-1114. CHEN, C.-K.; LO, C.-C.; LIAO, Y.-X. Optimal lot size with learning consideration on an imperfect production system with allowable shortages. International Journal 
of Production Economics, v. 113, n. 1, p. 459-469, 2008. http://dx.doi.org/10.1016/j.ijpe.2007.03.025

CHIU, H. N.; CHEN, H. M. An optimal algorithm for solving the dynamic lot-sizing model with learning and forgetting in setups and production. International Journal of Production Economics, v. 95, n. 2, p. 179-193, 2005. http://dx.doi.org/10.1016/j.ijpe.2003.12.001

COHEN, Y. et al. Allocation of work to the stations of an assembly line with buffers between stations and three general learning patterns. International Journal Intelligent Systems Technologies and Applications, v. 4, n. 1-2, p. 123-140, 2008a. http://dx.doi.org/10.1504/ IJISTA.2008.016362

COHEN, Y.; VITNER, G.; SARIN, S. Work allocation to stations with varying learning slopes and without buffers. European Journal of Operational Research, v. 184, n. 2, p. 797-801, 2008b. http://dx.doi.org/10.1016/j. ejor.2006.11.021

COHEN, Y.; VITNER, G.; SARIN, S. C. Optimal allocation of work in assembly lines for lots with homogenous learning. European Journal of Operational Research, v. 168, n. 3, p. 922-931, 2006. http://dx.doi.org/10.1016/j. ejor.2004.07.037

CONLEY, P. Experience Curves as a planning tool. IEEE Spectrum, v. 7, n. 6, p. 63-68, 1970. http://dx.doi. org/10.1109/MSPEC.1970.5213421

COROMINAS, A.; OLIVELLA, J.; PASTOR, R. A model for the assignment of a set of tasks when work performance depends on experience of all tasks involved. International Journal of Production Economics, v. 126, n. 2, p. 335-340, 2010. http://dx.doi.org/10.1016/j. ijpe.2010.04.012

CUNNINGHAM, B. J.; EBERLE, T. A guide to job enrichment and redesign. Personnel, v. 67, n. 2, p. 56-61, 1990.

DAVIS, L. E.; TAYLOR, J. C. Design of Jobs. 2. ed. Santa Mônica: Goodyear Publishing Company, 1979.

DE WIT, M. et al. Competition between biofuels: Modeling technological learning and cost reductions over time. Biomass and Bioenergy, v. 34, n. 2, p. 203-217, 2010. http://dx.doi.org/10.1016/j.biombioe.2009.07.012

DEMEESTER, L. L.; QI, M. Managing learning resources for consecutive product generations. International Journal of Production Economics, v. 95, n. 2, p. 265-283, 2005. http://dx.doi.org/10.1016/j.ijpe.2004.01.005

EREN, T. A bicriteria parallel machine scheduling with a learning effect of setup and removal times. Applied Mathematical Modelling, v. 33, n. 2, p. 11411150, 2009. http://dx.doi.org/10.1016/j.apm.2008.01.010

EREN, T.; GÜNER, E. Minimizing total tardiness in a scheduling problem with a learning effect. Applied Mathematical Modelling, v. 31, n. 7, p. 13511361, 2007. http://dx.doi.org/10.1016/j.apm.2006.03.030

FIORETTI, G. The organizational learning curve. European Journal of Operational Research, v. 177, n. 3, p. 13751384, 2007. http://dx.doi.org/10.1016/j.ejor.2005.04.009

FIORETTI, G. From Men and Machines to the Organizational Learning Curve. Social Science Research Network, 2009. Disponível em: <http://ssrn. com/abstract=1524332 . Acesso em: 09 jan. 2012.
FISK, J.; BALLOU, R. Production lot sizing under a learning effect. IIE Transactions, v. 14, n. 4, p. 251-264, 1982.

GUIMARÃES, L. B.; ANZANELLO, M. J.; RENNER, J. S. A learning curve-based method to implement multifunctional work teams in the Brazilian footwear sector. Applied Ergonomics, v. 43, n. 2, 2012.

HO, J.-W.; HUANG, Y.-S. A study on customer involvement in final assembly for DIY products. Computers \& Industrial Engineering, v. 56, n. 1, p. 19-27, 2009. http://dx.doi.org/10.1016/j.cie.2008.03.008

HSU, C.-J.; KUO, W.-H.; YANG, D.-L. Unrelated parallel machine scheduling with past-sequence-dependent setup times and learning effects. Applied Mathematical Modelling, v. 35, n. 3, p. 1492-1496, 2011. http:// dx.doi.org/10.1016/j.apm.2010.09.026

ISHIKAWA, K. Controle de qualidade total - à maneira japonesa. 2. ed. Rio de Janeiro: Campus, 1993.

ITTNER, C. D.; NAGAR, V.; RAJAN, M. V. An empirical examination of dynamic quality-based learning models. Management Science, v. 47, n. 4, p. 563-578, 2001. http://dx.doi.org/10.1287/mnsc.47.4.563.9831

JABER, M. Y.; BONNEY, M. Production breaks and the learning curve: The forgetting phenomenon. Applied Mathematical Modelling, v. 20, n. 20, p. 162-169, 1996a. http://dx.doi.org/10.1016/0307-904X(95)00157-F

JABER, M. Y.; BONNEY, M. Optimal lot sizing under learning considerations: The bounded learning case. Applied Mathematical Modelling, v. 20 , n. 10 , p. $750-755,1996$ b. http://dx.doi. org/10.1016/0307-904X(96)00072-8

JABER, M. Y.; BONNEY, M. The economic manufacture/ order quantity (EMQ/EOQ) and the learning curve: Past, present, and future. International Journal of Production Economics, v. 59, n. 1-3, p. 93-102, 1999. http://dx.doi.org/10.1016/S0925-5273(98)00027-9

JABER, M. Y.; BONNEY, M. Economic lot sizing with learning and continuous time discounting: Is it significant? International Journal of Production Economics, v. 71, n. 1-3, p. 135-143, 2001. http://dx.doi.org/10.1016/ S0925-5273(00)00113-4

JABER, M. Y.; BONNEY, M. Lot sizing with learning and forgetting in set-ups and in product quality. International Journal of Production Economics, v. 83 , n. 1, p. 95-111, 2003. http://dx.doi.org/10.1016/ S0925-5273(02)00322-5

JABER, M. Y.; BONNEY, M. Economic manufacture quantity (EMQ) model with lot-size dependent learning and forgetting rates. International Journal of Production Economics, v. 108, n. 1-2, p. 359-367, 2007. http://dx.doi.org/10.1016/j.ijpe.2006.12.020

JABER, M. Y.; EL SAADANY, A. M. A. An economic production and remanufacturing model with learning effects. International Journal of Production Economics, v. 131, n. 1, p. 115-127, 2011. http:// dx.doi.org/10.1016/j.ijpe.2009.04.019

JABER, M. Y.; GUIFFRIDA, A. L. Learning curves for processes generating defects requiring reworks. European Journal of Operational Research, v. 159, n. 3, p. 663-672, 2004. http://dx.doi.org/10.1016/ S0377-2217(03)00436-3 
JABER, M. Y.; BONNEY, M.; MOUALEK, I. Lot sizing with learning, forgetting and entropy cost. International Journal of Production Economics, v. 118, n. 1, p. 19-25, 2009a. http://dx.doi.org/10.1016/j. ijpe.2008.08.006

JABER, M. Y. et al. Entropic order quantity (EnOQ) model for deteriorating items. Applied Mathematical Modelling, v. 33, n. 1, p. 564-578, 2009b. http://dx.doi. org/10.1016/j.apm.2007.11.028

JABER, M. Y.; GOYAL, S. K.; IMRAN, M. Economic production quantity model for items with imperfect quality subject to learning effects. International Journal of Production Economics, v. 115, n. 1, p. 143- 150, 2008. http://dx.doi.org/10.1016/j.ijpe.2008.05.007

JABER, M. Y.; SIKSTRÖM, S. A numerical comparison of three potential learning and forgetting models. International Journal Production Economics, v. 92, n. 3, p. 281-294, 2004. http://dx.doi.org/10.1016/j. ijpe.2003.10.019

JANIAK, A. et al. Solution algorithms for the makespan minimization problem with the general learning model. Computers \& Industrial Engineering, v. 56, n. 4, p. 1301-1308, 2009. http://dx.doi.org/10.1016/j. cie.2008.07.019

KAHOULI-BRAHMI, S. Technological learning in energyenvironment-economy modelling: A survey. Energy Policy, v. 36, n. 1, p. 138-162, 2008. http://dx.doi. org/10.1016/j.enpol.2007.09.001

KEACHIE, E. C.; FONTANA, R. J. Production lot sizing under a learning effect. Management Science, v. 13, n. 2, p. 102-108, 1966. http://dx.doi.org/10.1287/ mnsc. 13.2.B102

KHAN, M.; JABER, M. Y.; WAHAB, M. I. Economic order quantity model for items with imperfect quality with learning in inspection. International Journal of Production Economics, v. 124, n. 1, p. 87-96, 2010. http://dx.doi.org/10.1016/j.ijpe.2009.10.011

KNECHT, G. Costing, Technological Growth and Generalized Learning Curves. Operations Research Quart, v. 25, n. 3, p. 487-491, 1974.

KOULAMAS, C.; KYPARISIS, G. J. Single-machine scheduling problems with past-sequence-dependent setup times. European Journal of Operational Research, v. 187, n. 3, p. 1045-1049, 2008. http:// dx.doi.org/10.1016/j.ejor.2006.03.066

KUO, W.-H.; YANG, D.-L. Single machine scheduling with past-sequence-dependent setup times and learning effects. Information Processing Letters, v. 102, n. 1, p. 22-26, 2007. http://dx.doi.org/10.1016/j. ipl.2006.11.002

LEE, W.-C. Scheduling with general position-based learning curves. Information Sciences, v. 181, n. 24, p. 55155522, 2011. http://dx.doi.org/10.1016/j.ins.2011.07.051

LEE, W.-C.; LAI, P.-J. Scheduling problems with general effects of deterioration and learning. Information Sciences, v. 181, n. 6, p. 1164-1170, 2011. http://dx.doi. org/10.1016/j.ins.2010.11.026

LEE, W.-C.; WU, C.-C. Minimizing total completion time in a two-machine flowshop with a learning effect. International Journal Production Economics, v. 88 , n. 1, p. 85-93, 2004. http://dx.doi.org/10.1016/ S0925-5273(03)00179-8

LEITE, M. O.; POSSAMAI, O. A Utilização das Curvas de Aprendizado no Planejamento da Construção Civil. In: ENCONTRO NACIONAL DE ENGENHARIA DE PRODUÇÃO, 21., 2001. Anais... Santa Catarina, 2001. CD-ROM.

LEVY, F. Adaptation in the Production Process. Management Science, v. 11, n. 6, p. 136-154, 1965. http://dx.doi.org/10.1287/mnsc.11.6.B136

LI, G.; RAJAGOPALAN, S. The impact of quality on learning. Journal of Operations Management, v. 15, n. 3, p. 181-191, 1997. http://dx.doi.org/10.1016/ S0272-6963(97)00003-X

LOPES, J. D. Análise e Otimização de Sequenciamento de Produção de uma Empresa de Médio Porte de Embalagens Plásticas. 2008. Monografia (Trabalho de Conclusão de Curso de Engenharia de Produção)Universidade Federal de Juiz de Fora, Juiz de Fora, 2008.

MANI, V.; CHANG, P. C.; CHEN, S. H. Bi-criteria single machine scheduling problem with a learning effect: Aneja-Nair method to obtain the set of optimal sequences. Computers and Mathematics with Applications, v. 58, n. 1, p. 39-47, 2009. http://dx.doi.org/10.1016/j. camwa.2009.01.042

MAZUR, J. E.; HASTIE, R. Learning as Accumulation: a Reexamination of the Learning Curve. Psychological Bulletin, v. 85, n. 6, p. 1256-1274, 1978. PMid:734012. http://dx.doi.org/10.1037/0033-2909.85.6.1256

MORRISON, J. B. Putting the learning curve in context. Journal of Business Research, v. 61, n. 11, p. 1182-1190, 2008. http://dx.doi.org/10.1016/j. jbusres.2007.11.009

NADEAU, M.-C. et al. A dynamic process-based cost modeling approach to understand learning effects in manufacturing. International Journal of Production Economics, v. 128, n. 1, p. 223-234, 2010. http://dx.doi. org/10.1016/j.ijpe.2010.07.016

NEMBHARD, D. A.; OSOTHSILP, N. Task complexity effects on between-individual learning/forgetting variability. International Journal of Industrial Ergonomics, v. 29, n. 5, p. 297-306, 2002. http:// dx.doi.org/10.1016/S0169-8141(01)00070-1

NEMBHARD, D. A.; UZUMERI, M. V. An Individual-Based Description of Learning within an Organization. IEEE Transactions on Engineering Management, v. 47, n. 3, p. 370-378, 2000. http://dx.doi.org/10.1109/17.865905

NEMET, G. F. Beyond the learning curve: factors influencing cost reductions in photovoltaics. Energy Policy, v. 34, n. 17 , p. 3218-3232, 2006. http://dx.doi.org/10.1016/j. enpol.2005.06.020

NEUHAUS, M.; BUNKE, H. Automatic learning of cost functions for graph edit distance. Information Sciences, v. 177, n. 1, p. 239-247, 2007. http://dx.doi. org/10.1016/j.ins.2006.02.013

NGWENYAMA, O.; GUERGACHI, A.; McLAREN, T. Using the learning curve to maximize IT productivity: A decision analysis model for timing software upgrades. International Journal of Production Economics, v. 105, n. 2, p. 524-535, 2007. http://dx.doi.org/10.1016/j. ijpe.2006.02.013 
OTERO, L. D. et al. A systematic approach for resource allocation in software projects. Computers \& Industrial Engineering, v. 56, n. 4, p. 1333-1339, 2009. http:// dx.doi.org/10.1016/j.cie.2008.08.002

PENDHARKAR, P. C.; SUBRAMANIAN, G. H. An empirical study of ICASE learning curves and probability bounds for software development effort. European Journal of Operational Research, v. 183, n. 3, p. 10861096, 2007. http://dx.doi.org/10.1016/j.ejor.2005.07.029

PEREIRA, W. C.; SUSLICK, S. B. Modelo de previsão do custo de mineração pelo sistema de curvas de aprendizado. Revista Escola de Minas, v. 56, n. 2, p. 139-143, 2003. http://dx.doi.org/10.1590/S0370-44672003000200013

PLAZA, M.; ROHLF, K. Learning and performance in ERP implementation projects: A learning-curve model for analyzing and managing consulting costs. International Journal of Production Economics, v. 115 , n. 1, p. 72- 85, 2008. http://dx.doi.org/10.1016/j. ijpe.2008.05.005

PLAZA, M.; NGWENYAMA, O. K.; ROHLF, K. A comparative analysis of learning curves: Implications for new technology implementation management. European Journal of Operational Research, v. 200, n. 2, p. 518-528, 2010. http://dx.doi.org/10.1016/j. ejor.2009.01.010

PROCHNO, P. Learning Curve? Which One? Brazilian Administration Review, v. 1, n. 1, p. 53-66, 2004. http://dx.doi.org/10.1590/S1807-76922004000100005

PRUETT, M.; THOMAS, H. Experience-based learning in innovation and production. R\&D Management, v. 38, n. 2, p. 141-153, 2008. http://dx.doi. org/10.1111/j.1467-9310.2008.00504.x

RACHAMADUGU, R.; SCHRIBER, T. J. Optimal and heuristic policies for lot sizing with learning in setups. Journal of Operations Management, v. 13, n. 3 , p. 229-245, 1995. http://dx.doi. org/10.1016/0272-6963(95)00024-M

RACHAMADUGU, R.; TAN, C. L. Policies for lot sizing with setup learning. International Journal of Production Economics, v. 48, n. 2, p. 157-165, 1997. http://dx.doi.org/10.1016/S0925-5273(97)82105-6

SCHOOTS, K.; KRAMER, G. J.; van der ZWAAN, B.C.C. Technology learning for fuel cells: An assessment of past and potential cost reductions. Energy Policy, v. 38, n. 6, p. 2887-2897, 2010. http://dx.doi.org/10.1016/j. enpol.2010.01.022

SHAFER, S. M.; NEMBHARD, D. A.; UZUMERI, M. V. The Effects of Worker Learning, Forgetting, and Heterogeneity on Assembly Line Productivity. Management Science, v. 47, n. 12, p. 1639-1653, 2001. http://dx.doi.org/10.1287/mnsc.47.12.1639.10236

SMUNT, T. L.; WATTS, C. A. Improving operations planning with learning curves: overcoming the pitfalls of 'messy' shop floor data. Journal of Operations Management, v. 21, n. 1, p. 93-107, 2003. http://dx.doi.org/10.1016/ S0272-6963(02)00088-8

STAN, M.; VERMEULEN, F. Selection at the Gate: Difficult Cases, Spillovers, and Organizational Learning. In: SUMMER CONFERENCE 2010 ON OPENING UP INNOVATION: STRATEGY, ORGANIZATION
AND TECHNOLOGY, 2010, Londres. Proceedings... Londres, 2010.

SZWARCFITER, C.; DALCOL, P. R. Economias de Escala e de Escopo: Desmistificando alguns Aspectos da Transição. Produção, v. 7, n. 2, p. 117-129, 1997. http://dx.doi.org/10.1590/S0103-65131997000200001

TARNANIDIS, J.; PAPATHANASIOU, J.; FILAKIS, P. Learning Curves As a Tool Of Enterprises Development. Advanced Modeling and Optimization, v. 8, n. 2, p. 209-216, 2006.

TENG, J.-T.; THOMPSON, G. L. Optimal strategies for general price-quality decision models of new products with learning production costs. European Journal of Operational Research, v. 93, n. 3, p. 476-489, 1996. http://dx.doi.org/10.1016/0377-2217(96)00028-8

TOKSARI, M. D. et al. Simple and U-type assembly line balancing problems with a learning effect. Applied Mathematical Modelling, v. 32, n. 12, p. 29542961, 2008. http://dx.doi.org/10.1016/j.apm.2007.10.007

TOKSARI, M. D. et al. Assembly line balancing problem with deterioration tasks and learning effect. Expert Systems with Applications, v. 37, n. 2, p. 12231228, 2010. http://dx.doi.org/10.1016/j.eswa.2009.06.005

TOWILL, D. R. Forecasting Learning Curves. International Journal of Forecasting, v. 6, n. 1, p. 25-38, 1990. http:// dx.doi.org/10.1016/0169-2070(90)90095-S

TSUCHIYA, H.; KOBAYASHI, O. Mass production cost of PEM fuel cell by learning curve. International Journal of Hydrogen Energy, v. 29, n. 10, p. 985-990, 2004. http://dx.doi.org/10.1016/j.ijhydene.2003.10.011

WAHAB, M. I.; JABER, M. Y. Economic order quantity model for items with imperfect quality, different holding costs, and learning effects: A note. Computers \& Industrial Engineering, v. 58, n. 1, p. 186-190, 2010. http://dx.doi.org/10.1016/j.cie.2009.07.007

WANG, J.-B.; SUN, L.; SUN, L. Single machine scheduling with exponential sum-of-logarithm-processing-times based learning effect. Applied Mathematical Modelling, v. 34, n. 10, p. 2813-2819, 2010. http:// dx.doi.org/10.1016/j.apm.2009.12.015

WEITZEL, L.; ROVERE, E.L.; CUNHA, R. As demandas do novo modelo institucional do setor elétrico brasileiro. In: CONGRESSO BRASILEIRO DE PLANEJAMENTO ENERGÉTICO, 5.; 2006, Brasília. Anais... Brasília: Sociedade Brasileira de Planejamento Energético, 2006. CD-ROM.

WIERSMA, E. Conditions That Shape the Learning Curve: Factors That Increase the Ability and Opportunity to Learn. Management Science, v. 53, n. 12, p. 19031915, 2007. http://dx.doi.org/10.1287/mnsc.1070.0733

WRIGHT, T. P. Factors affecting the cost of airplanes. Journal of the Aeronautical Sciences, v. 3, n. 4, p. 122-128, 1936.

WU, C.-C.; YIN, Y.; CHENG, S.-R. Some single-machine scheduling problems with a truncation learning effect. Computers \& Industrial Engineering, v. 60, n. 4, p. 790-795, 2011. http://dx.doi.org/10.1016/j. cie.2011.01.016

YANG, L.; WANG, Y.-R.; PAI, S. On-line SPC with consideration of learning curve. Computers \& Industrial 
Engineering, v. 57, n. 3, p. 1089-1095, 2009. http:// dx.doi.org/10.1016/j.cie.2009.04.015

YANG, S.-J. Group scheduling problems with simultaneous considerations of learning and deterioration effects on a single-machine. Applied Mathematical Modelling, v. 35, n. 8, p. 4008-4016, 2011. http://dx.doi.org/10.1016/j. apm.2011.02.024

YELLE, L. E. The Learning Curve: Historical Review and Comprehensive Survey. Decision Science, v. 10, n. 2 , p. 302-328, 1979. http://dx.doi. org/10.1111/j.1540-5915.1979.tb00026.X

YIN, Y.; XU, D. Some single-machine scheduling problems with general effects of learning and deterioration.
Computers and Mathematics with Applications, v. 61, n. 1, p. 100-108, 2011. http://dx.doi.org/10.1016/j. camwa.2010.10.036

YIN, Y.; XU, D.; WANG, J. Single-machine scheduling with a general sum-of-actual-processing-times-based and job-position-based learning effect. Applied Mathematical Modelling, v. 34, n. 10, p. 3623 3630, 2010. http://dx.doi.org/10.1016/j.apm.2010.03.011 YOUNG II, W. A.; MASEL, D. T.; JUDD, R. P. A matrixbased methodology for determining a part family's learning rate. Computers \& Industrial Engineering, v. 54, n. 3, p. 3, 2008. http://dx.doi.org/10.1016/j. cie.2007.08.002 